PROCEEDINGS OF THE

AMERICAN MATHEMATICAL SOCIETY

Volume 135, Number 1, January 2007, Pages 21-29

S 0002-9939(06)08464-4

Article electronically published on June 22, 2006

\title{
ON THE STRUCTURE OF QUANTUM PERMUTATION GROUPS
}

\author{
TEODOR BANICA AND SERGIU MOROIANU
}

(Communicated by Martin Lorenz)

\begin{abstract}
The quantum permutation group of the set $X_{n}=\{1, \ldots, n\}$ corresponds to the Hopf algebra $A_{\text {aut }}\left(X_{n}\right)$. This is an algebra constructed with generators and relations, known to be isomorphic to $\mathbb{C}\left(S_{n}\right)$ for $n \leq 3$, and to be infinite dimensional for $n \geq 4$. In this paper we find an explicit representation of the algebra $A_{\text {aut }}\left(X_{n}\right)$, related to Clifford algebras. For $n=4$ the representation is faithful in the discrete quantum group sense.
\end{abstract}

\section{INTRODUCTION}

A general theory of unital Hopf $\mathbb{C}^{*}$-algebras was developed by Woronowicz in [11, [12, 13. The main results are the existence of the Haar functional, an analogue of Peter-Weyl theory and of Tannaka-Krein duality, and explicit formulae for the square of the antipode. As for examples, these include algebras of continuous functions on compact groups, $q$-deformations of them with $q>0$, and $\mathbb{C}^{*}$-algebras of discrete groups.

Of particular interest is the algebra $A_{a u t}\left(X_{n}\right)$ constructed by Wang in [9]. This is the universal Hopf $\mathbb{C}^{*}$-algebra coacting on the set $X_{n}=\{1, \ldots, n\}$. In other words, the compact quantum group associated to it is a kind of analogue of the symmetric group $S_{n}$.

The algebra $A_{\text {aut }}\left(X_{n}\right)$ is constructed with generators and relations. There are $n^{2}$ generators, labeled $u_{i j}$ with $i, j=1, \ldots, n$. The relations are those making $u$ a magic biunitary matrix. This means that all coefficients $u_{i j}$ are projections, and on each row and each column of $u$ these projections are mutually orthogonal, and sum up to 1 . The comultiplication is given by $\Delta\left(u_{i j}\right)=\sum u_{i k} \otimes u_{k j}$, and the fundamental coaction is given by $\alpha\left(\delta_{i}\right)=\sum u_{j i} \otimes \delta_{j}$.

For $n=1,2,3$ the canonical quotient map $A_{\text {aut }}\left(X_{n}\right) \rightarrow \mathbb{C}\left(S_{n}\right)$ is an isomorphism. For $n \geq 4$ the algebra $A_{\text {aut }}\left(X_{n}\right)$ is infinite dimensional, and just a few things are known about it. Its irreducible corepresentations are classified in [3], with the conclusion that their fusion rules coincide with those for irreducible representations of $S O(3)$, independently of $n \geq 4$. In [10] Wang proves that the compact quantum

Received by the editors November 24, 2004 and, in revised form, July 28, 2005.

2000 Mathematics Subject Classification. Primary 16W30; Secondary 81R50.

Key words and phrases. Hopf algebra, magic biunitary matrix, inner faithful representation.

The second author was partially supported by the Marie Curie grant MERG-CT-2004-006375 funded by the European Commission, and by a CERES contract (2004).

(C)2006 American Mathematical Society Reverts to public domain 28 years from publication 
group associated to $A_{\text {aut }}\left(X_{n}\right)$ with $n \geq 4$ is simple. In $[3$ it is shown that the discrete quantum group associated to $A_{\text {aut }}\left(X_{n}\right)$ with $n \geq 5$ is not amenable. Various quotients of $A_{\text {aut }}\left(X_{n}\right)$, corresponding to quantum symmetry groups of polyhedra, colored graphs etc., are studied in [4] by using planar algebra techniques.

These results certainly bring some light on the structure of $A_{\text {aut }}\left(X_{n}\right)$. However, for $n \geq 4$ this remains an abstract algebra, constructed with generators and relations.

In this paper we find an explicit representation of $A_{\text {aut }}\left(X_{n}\right)$. The construction works when $n$ is a power of 2 , and uses a magic biunitary matrix related to Clifford algebras. For $n=4$ the representation is inner faithful, in the sense that the corresponding unitary representation of the discrete quantum group associated to $A_{\text {aut }}\left(X_{4}\right)$ is faithful.

As a conclusion, there might be a geometric interpretation of Hopf algebras of type $A_{\text {aut }}\left(X_{n}\right)$. We should mention here that for the algebra $A_{\text {aut }}(X)$ with an $X$ finite graph, such an interpretation would be of real help, for instance in computing fusion rules.

\section{Magic Biunitary matrices}

Let $A$ be a unital $\mathbb{C}^{*}$-algebra. That is, we have a unital algebra $A$ over the field of complex numbers $\mathbb{C}$, with an antilinear antimultiplicative map $a \rightarrow a^{*}$ satisfying $a^{* *}=a$, and with a Banach space norm satisfying $\left\|a^{*} a\right\|=\|a\|^{2}$.

A projection is an element $p \in A$ satisfying $p^{2}=p^{*}=p$. Two projections $p, q$ are said to be orthogonal when $p q=0$. A partition of unity in $A$ is a finite set of projections, which are mutually orthogonal, and sum up to 1 .

Definition 1.1. A matrix $v \in M_{n}(A)$ is called magic biunitary if all its rows and columns are partitions of the unity of $A$.

A magic biunitary is indeed a biunitary, in the sense that both $v$ and its transpose $v^{t}$ are unitaries. The other word - magic - comes from a vague similarity with magic squares.

The basic example comes from the symmetric group $S_{n}$. Consider the sets of permutations $\left\{\sigma \in S_{n} \mid \sigma(j)=i\right\}$. When $i$ is fixed and $j$ varies, or vice versa, these sets form partitions of $S_{n}$. Thus their characteristic functions $v_{i j} \in \mathbb{C}\left(S_{n}\right)$ form a magic biunitary.

Of particular interest is the "universal" magic biunitary matrix. This has coefficients in the universal algebra $A_{\text {aut }}\left(X_{n}\right)$ constructed by Wang in [9].

Definition 1.2. $A_{\text {aut }}\left(X_{n}\right)$ is the universal $\mathbb{C}^{*}$-algebra generated by $n^{2}$ elements $u_{i j}$, subject to the magic biunitarity condition.

In other words, we have the following universal property. For any magic biunitary matrix $v \in M_{n}(A)$ there is a morphism of $\mathbb{C}^{*}$-algebras $A_{\text {aut }}\left(X_{n}\right) \rightarrow A$ mapping $u_{i j} \rightarrow v_{i j}$.

A more elaborate version of this property, to be discussed now, states that $A_{\text {aut }}\left(X_{n}\right)$ is a Hopf $\mathbb{C}^{*}$-algebra, whose underlying quantum group is a kind of analogue of $S_{n}$.

The following definition is due to Woronowicz [13].

Definition 1.3. A unital Hopf $\mathbb{C}^{*}$-algebra is a unital $\mathbb{C}^{*}$-algebra $A$, together with a morphism of $\mathbb{C}^{*}$-algebras $\Delta: A \rightarrow A \otimes A$, subject to the following conditions. 
(i) Coassociativity condition: $(\Delta \otimes i d) \Delta=(i d \otimes \Delta) \Delta$.

(ii) Cocancellation condition: $\Delta(A)(1 \otimes A)$ and $\Delta(A)(A \otimes 1)$ are dense in $A \otimes A$.

The basic example is the algebra $\mathbb{C}(G)$ of continuous functions on a compact group $G$, with $\Delta(\varphi):(g, h) \rightarrow \varphi(g h)$. Here coassociativity of $\Delta$ follows from associativity of the multiplication of $G$, and cocancellation in $\mathbb{C}(G)$ follows from cancellation in $G$.

Another example is the group algebra $\mathbb{C}^{*}(\Gamma)$ of a discrete group $\Gamma$. This is obtained from the usual group algebra $\mathbb{C}[\Gamma]$ by a standard completion procedure. The comultiplication is defined on generators $g \in \Gamma$ by the formula $\Delta(g)=g \otimes g$.

In general, associated to a Hopf $\mathbb{C}^{*}$-algebra $A$ are a compact quantum group $G$ and a discrete quantum group $\Gamma$, according to the heuristic formula $A=\mathbb{C}(G)=$ $\mathbb{C}^{*}(\Gamma)$.

Definition 1.4. A coaction of $A$ on a finite set $X$ is a morphism of $\mathbb{C}^{*}$-algebras $\alpha: \mathbb{C}(X) \rightarrow \mathbb{C}(X) \otimes A$, subject to the following conditions:

(i) Coassociativity condition: $(\alpha \otimes i d) \alpha=(i d \otimes \Delta) \alpha$.

(ii) Natural condition: $(\Sigma \otimes i d) v=\Sigma()$.1 , where $\Sigma(\varphi)$ is the sum of values of $\varphi$.

The basic example is with a group $G$ of permutations of $X$. Consider the action map $a: X \times G \rightarrow X$, given by $a(i, \sigma)=\sigma(i)$. The formula $\alpha \varphi=\varphi a$ defines a morphism of $\mathbb{C}^{*}$-algebras $\alpha: \mathbb{C}(X) \rightarrow \mathbb{C}(X \times G)$. This can be regarded as a coaction of $\mathbb{C}(G)$ on $X$.

In general, coactions of $A$ can be thought of as coming from actions of the underlying compact quantum group $G$. With this interpretation, the natural condition says that the action of $G$ must preserve the counting measure on $X$. This assumption cannot be dropped.

The following fundamental result is due to Wang [9].

Theorem 1.1. (i) $A_{\text {aut }}\left(X_{n}\right)$ is a Hopf $\mathbb{C}^{*}$-algebra, with comultiplication $\Delta\left(u_{i j}\right)=$ $\sum u_{i k} \otimes u_{k j}$.

(ii) The linear map $\alpha\left(\delta_{j}\right)=\sum \delta_{i} \otimes u_{j i}$ is a coaction of $A_{a u t}\left(X_{n}\right)$ on $X_{n}=$ $\{1, \ldots, n\}$.

(iii) $A_{\text {aut }}\left(X_{n}\right)$ is the universal Hopf $\mathbb{C}^{*}$-algebra coacting on $X_{n}$.

The idea for proving (i) is that we can define $\Delta$ by using the universal property of $A_{\text {aut }}\left(X_{n}\right)$. Coassociativity is clear, and cocancellation follows from a result of Woronowicz in [13], stating that this is automatic whenever there is a counit and an antipode. But these can be defined by $\varepsilon\left(u_{i j}\right)=\delta_{i j}$ and $S\left(u_{i j}\right)=u_{j i}$, once again by using universality of $A_{a u t}\left(X_{n}\right)$.

We know that the compact quantum group $G_{n}$ associated to $A_{a u t}\left(X_{n}\right)$ is a kind of quantum analogue of the symmetric group $S_{n}$. In particular there should be an inclusion $S_{n} \subset G_{n}$. Here is the exact formulation of this observation; see Wang [9] for details.

Proposition 1.1. There is a Hopf $\mathbb{C}^{*}$-algebra morphism $\pi_{n}: A_{\text {aut }}\left(X_{n}\right) \rightarrow \mathbb{C}\left(S_{n}\right)$, mapping the generators $u_{i j}$ to the characteristic functions of the sets $\left\{\sigma \in S_{n}\right.$ | $\sigma(j)=i\}$.

The question is now whether $\pi_{n}$ is an isomorphism or not. For instance a $2 \times 2$ magic biunitary must be of the following special form, where $p$ is a projection:

$$
\left(\begin{array}{cc}
p & 1-p \\
1-p & p
\end{array}\right)
$$


The algebra generated by $p$ is canonically isomorphic to $\mathbb{C}^{2}$ if $p \neq 0,1$, and to $\mathbb{C}$ if not. Thus the universal algebra $A_{\text {aut }}\left(X_{2}\right)$ is isomorphic to $\mathbb{C}^{2}$, and $\pi_{2}$ is an isomorphism.

The map $\pi_{3}$ is an isomorphism as well; see [4 for a proof.

At $n=4$ we have the following example of a magic biunitary matrix:

$$
\left(\begin{array}{cccc}
p & 1-p & 0 & 0 \\
1-p & p & 0 & 0 \\
0 & 0 & q & 1-q \\
0 & 0 & 1-q & q
\end{array}\right)
$$

We can choose the projections $p, q$ such that the algebra $\langle p, q\rangle$ they generate is infinite dimensional and not commutative. It follows that $A_{\text {aut }}\left(X_{4}\right)$ is infinite dimensional and not commutative as well, so $\pi_{4}$ cannot be an isomorphism.

Proposition 1.2. For $n \geq 4$ the algebra $A_{a u t}\left(X_{n}\right)$ is infinite dimensional and not commutative. In particular $\pi_{n}$ is not an isomorphism.

This follows by gluing an identity matrix of size $n-4$ to the above $4 \times 4$ matrix.

There is a quantum group interpretation here. Consider the compact and discrete quantum groups defined by the formula $A_{\text {aut }}\left(X_{4}\right)=\mathbb{C}\left(G_{4}\right)=\mathbb{C}^{*}\left(\Gamma_{4}\right)$. When $p, q$ are free the surjective morphism of $\mathbb{C}^{*}$-algebras $A_{\text {aut }}\left(X_{4}\right) \rightarrow\langle p, q\rangle$ can be thought of as coming from a surjective morphism of discrete quantum groups $\Gamma_{4} \rightarrow \mathbb{Z}_{2} * \mathbb{Z}_{2}$. This makes it clear that $\Gamma_{4}$ is infinite. Now $G_{4}$ being the Pontrjagin dual of $\Gamma_{4}$, it must be infinite as well.

See Bichon [5], Wang 9], 10] and Banica [4] for further speculations on this subject.

\section{INNER FAITHFUL REPRESENTATIONS}

We would like to find an explicit representation of $A_{a u t}\left(X_{n}\right)$. As with any Hopf $\mathbb{C}^{*}$-algebra, there is a problem here, because there are two notions of faithfulness.

Consider for instance a discrete subgroup $\Gamma$ of the unitary group $U(n)$. The inclusion $\Gamma \subset U(n)$ can be regarded as a unitary group representation $\Gamma \rightarrow U(n)$, and we get a $\mathbb{C}^{*}$-algebra representation $\mathbb{C}^{*}(\Gamma) \rightarrow M_{n}(\mathbb{C})$. This latter representation is far from being faithful: for instance its kernel is infinite dimensional, hence nonempty, when $\Gamma$ is an infinite group. However, the representation $\mathbb{C}^{*}(\Gamma) \rightarrow M_{n}(\mathbb{C})$ must be "inner faithful" in some Hopf $\mathbb{C}^{*}$-algebra sense, because the representation $\Gamma \rightarrow U(n)$ it comes from is faithful.

So, we are led to the following question. Let $H$ be a unital Hopf $\mathbb{C}^{*}$-algebra, and let $\pi: H \rightarrow A$ be a morphism of $\mathbb{C}^{*}$-algebras. If $\Gamma$ is the discrete quantum group associated to $H$ we know that $\pi$ corresponds to a unitary representation $\pi_{i}: \Gamma \rightarrow U(A)$. The question is: when is $\pi$ inner faithful, meaning that $\pi_{i}$ is faithful?

A simple answer is obtained by using the formalism of Kustermans and Vaes [6]. Associated to $H$ is a von Neumann algebra $H_{v N}$, obtained by a certain completion procedure. Now coefficients of $\pi$ belong to the dual algebra $\widehat{H}_{v N}$, and we can say that $\pi$ is inner faithful if these coefficients generate $\widehat{H}_{v N}$. This notion is used by Vaes in [7, and a version of it is used by Wang in [9].

In this paper we use an equivalent definition from [2]. 
Definition 2.1. Let $H$ be a unital Hopf $\mathbb{C}^{*}$-algebra. A $\mathbb{C}^{*}$-algebra representation $\pi: H \rightarrow A$ is called inner faithful if the $*$-algebra generated by its coefficients is dense in $H_{a l g}^{*}$.

Here $H_{\text {alg }}$ is the dense $*$-subalgebra of $H$ consisting of "representative functions" on the underlying compact quantum group, constructed by Woronowicz in [13. This is a Hopf $*$-algebra in the usual sense. Its dual complex vector space $H_{\text {alg }}^{*}$ is a $*$-algebra, with multiplication $\Delta^{*}$ and involution $*^{*}$. Finally, coefficients of $\pi$ are the linear forms $\varphi \pi$ with $\varphi \in A^{*}$, and the density assumption is with respect to the weak topology on $H_{a l g}^{*}$. See e.g. the book of Abe [1] for Hopf algebras and [2] for details regarding this definition.

The main example is with a discrete group $\Gamma$. As expected, a representation $\mathbb{C}^{*}(\Gamma) \rightarrow A$ is inner faithful if and only if the corresponding unitary group representation $\Gamma \rightarrow U(A)$ is faithful. Some other examples are discussed in [2].

For how to use inner faithfulness see Vaes [7].

Definition 2.2. The character of a magic biunitary matrix $v \in M_{n}(A)$ is the sum of its diagonal entries $\chi(v)=v_{11}+v_{22}+\ldots+v_{n n}$.

The terminology comes from the case where $v=u$ is the universal magic biunitary matrix, with coefficients in $A=A_{a u t}\left(X_{n}\right)$. Indeed, the matrix $u$ is a corepresentation of $A_{\text {aut }}\left(X_{n}\right)$ in the sense of Woronowicz [11, and the element $\chi(u)$ is its character.

Lemma 2.1. Let $v \in M_{n}(A)$ be a magic biunitary matrix, with $n \geq 4$. Assume that there is a unital linear form $\varphi: A \rightarrow \mathbb{C}$ such that

$$
\varphi\left(\chi(v)^{k}\right)=\frac{1}{k+1}\left(\begin{array}{c}
2 k \\
k
\end{array}\right)
$$

for any $k$. Then the representation $\pi: A_{a u t}\left(X_{n}\right) \rightarrow A$ defined by $u_{i j} \rightarrow v_{i j}$ is inner faithful.

Proof. The numbers in the statement are the Catalan numbers, appearing as multiplicities in the representation theory of $S O(3)$. The result will follow from the following fact from [3]. The finite-dimensional irreducible corepresentations of $A_{\text {aut }}\left(X_{n}\right)$ can be arranged in a sequence $\left\{r_{k}\right\}$, such that their fusion rules are the same as those for representations of $S O(3)$ :

$$
r_{k} \otimes r_{s}=r_{|k-s|}+r_{|k-s|+1}+\ldots+r_{k+s} .
$$

Let $h: A_{\text {aut }}\left(X_{n}\right) \rightarrow \mathbb{C}$ be the Haar functional constructed by Woronowicz in 11]. Also consider the character of the fundamental corepresentation of $A_{\text {aut }}\left(X_{n}\right)$ :

$$
\chi(u)=u_{11}+u_{22}+\ldots+u_{n n} .
$$

The Poincaré series of $A_{\text {aut }}\left(X_{n}\right)$ is defined by the following formula:

$$
f(z)=\sum_{k=0}^{\infty} h\left(\chi(u)^{k}\right) z^{k} .
$$

By the above result, this is equal to the Poincaré series for $S O(3)$ :

$$
f(z)=\sum_{k=0}^{\infty} \frac{1}{k+1}\left(\begin{array}{c}
2 k \\
k
\end{array}\right) z^{k} .
$$


The assumption of the lemma says that the equality $\varphi \pi=h$ holds on all powers of $\chi(u)$. By linearity, this equality must hold on the algebra $\langle\chi(u)\rangle$ generated by $\chi(u)$. Now by positivity of $h$ it follows that the restriction of $\pi$ to this algebra $\langle\chi(u)\rangle$ is injective.

On the other hand, once again from fusion rules, we see that $\chi(u)$ generates the algebra of characters $A_{a u t}\left(X_{n}\right)_{\text {central }}$ constructed by Woronowicz in [1].

Summing up, we know that $\pi$ is faithful on $A_{\text {aut }}\left(X_{n}\right)_{\text {central }}$.

Now consider the "minimal model" construction in 2]. This is the factorisation of $\pi$ into a Hopf $\mathbb{C}^{*}$-algebra morphism $A_{\text {aut }}\left(X_{n}\right) \rightarrow H$, and an inner faithful representation $H \rightarrow A$ :

$$
A_{\text {aut }}\left(X_{n}\right) \rightarrow H \rightarrow A \text {. }
$$

Since $\pi$ is faithful on $A_{a u t}\left(X_{n}\right)_{\text {central }}$, so is the map on the left. By Woronowicz's analogue of the Peter-Weyl theory in [11, it follows that the map on the left is an isomorphism. Thus $\pi$ coincides with the map on the right, which is by definition inner faithful.

It is possible to reformulate this result by using notions from Voiculescu's free probability theory 8 . A non-commutative $\mathbb{C}^{*}$-probability space is a pair $(A, \varphi)$ consisting of a unital $\mathbb{C}^{*}$-algebra $A$ together with a positive unital linear form $\varphi: A \rightarrow \mathbb{C}$.

Associated to a self-adjoint element $x \in A$ is its spectral measure $\mu_{x}$. This is a probability measure on the spectrum of $x$, defined by the formula

$$
\varphi(f(x))=\int_{\mathbb{R}} f(t) d \mu_{x}(t) .
$$

This equality must hold for any continuous function $f$ on the spectrum of $x$. By density we can restrict our attention to polynomials $f \in \mathbb{C}[X]$, then by linearity it is enough to have this equality for monomials $f(t)=t^{k}$. We say that $\mu_{x}$ is uniquely determined by its moments,

$$
\varphi\left(x^{k}\right)=\int_{\mathbb{R}} t^{k} d \mu_{x}(t) .
$$

The following notion plays a central role in free probability. See [8], page 26.

Definition 2.3. An element $x$ in a non-commutative $\mathbb{C}^{*}$-probability space is called semicircular if its spectral measure is $d \mu_{x}(t)=(2 \pi)^{-1} \sqrt{4-t^{2}} d t$ on $[-2,2]$, and 0 elsewhere.

In terms of moments, we must have the following equalities, for any $k$ :

$$
\varphi\left(x^{k}\right)=\frac{1}{2 \pi} \int_{-2}^{2} t^{k} \sqrt{4-t^{2}} d t .
$$

The integral is 0 when $k$ is odd, and equal to a Catalan number when $k$ is even,

$$
\varphi\left(x^{2 k}\right)=\frac{1}{k+1}\left(\begin{array}{c}
2 k \\
k
\end{array}\right) .
$$

We get in this way a reformulation of the above lemma.

Theorem 2.1. A magic biunitary matrix whose character has the same spectral measure as the square of a semicircular element produces an inner faithful representation of Wang's algebra. 
The assumption $n \geq 4$ was removed, because it is superfluous. Indeed, for $n=1,2,3$ finite dimensionality of $A_{\text {aut }}\left(X_{n}\right)$ implies that the spectrum of any $\chi(v)$ is discrete.

\section{Geometric constructions}

Consider the Pauli matrices,

$$
1=\left(\begin{array}{cc}
1 & 0 \\
0 & 1
\end{array}\right), \quad i=\left(\begin{array}{cc}
i & 0 \\
0 & -i
\end{array}\right), \quad j=\left(\begin{array}{cc}
0 & 1 \\
-1 & 0
\end{array}\right), \quad k=\left(\begin{array}{ll}
0 & i \\
i & 0
\end{array}\right) .
$$

These satisfy the relations for quaternions $i^{2}=j^{2}=k^{2}=-1, i j=j i=-k$, etc.

To any $x \in S U(2)$ we associate the following matrix:

$$
\left(\begin{array}{l}
1 \\
i \\
j \\
k
\end{array}\right) x\left(\begin{array}{llll}
1 & i & j & k
\end{array}\right)=\left(\begin{array}{cccc}
x & x i & x j & x k \\
i x & i x i & i x j & i x k \\
j x & j x i & j x j & j x k \\
k x & k x i & k x j & k x k
\end{array}\right) .
$$

Each row and each column of this matrix is an orthogonal basis of $M_{2}(\mathbb{C}) \simeq \mathbb{C}^{4}$ with respect to the inner product

$$
\langle x, y\rangle=\frac{1}{2} \operatorname{Tr}\left(x y^{*}\right)
$$

since $i, j, k$ are skew-adjoints. Thus the matrix of corresponding orthogonal projections is a magic biunitary.

Theorem 3.1. There is an inner faithful representation

$$
\pi: A_{\text {aut }}\left(X_{4}\right) \rightarrow \mathbb{C}\left(S U(2), M_{4}(\mathbb{C})\right)
$$

mapping the universal $4 \times 4$ magic biunitary matrix to the $4 \times 4$ matrix

$$
v(x)=\left(\begin{array}{cccc}
P_{x} & P_{x i} & P_{x j} & P_{x k} \\
P_{i x} & P_{i x i} & P_{i x j} & P_{i x k} \\
P_{j x} & P_{j x i} & P_{j x j} & P_{j x k} \\
P_{k x} & P_{k x i} & P_{k x j} & P_{k x k}
\end{array}\right)
$$

where for $y \in S U(2)$ we denote by $P_{y}$ the orthogonal projection onto the space $\mathbb{C} y \subset$ $M_{2}(\mathbb{C})$, and we regard it as a continuous function of $y$, with values in $M_{2}\left(M_{2}(\mathbb{C})\right) \simeq$ $M_{4}(\mathbb{C})$.

Proof. We have to compute the character of $v=v(x)$ :

$$
\chi(v)=P_{x}+P_{i x i}+P_{j x j}+P_{k x k} .
$$

We make the convention that Greek letters designate quaternions in $\{1, i, j, k\}$. We decompose $x$ as a sum with real coefficients $x=\sum x_{\alpha} \alpha$. We have the following formula for $\chi(v)$ :

$$
\chi(v)=\sum_{\alpha} P_{\alpha x \alpha} .
$$

With the notations $\alpha \beta=(-1)^{N(\alpha, \beta)} \beta \alpha$ and $\alpha^{2}=(-1)^{N(\alpha)}$ we can compute $\alpha x \alpha$ :

$$
\alpha x \alpha=\sum_{\beta}(-1)^{N(\alpha, \beta)+N(\alpha)} x_{\beta} \beta
$$


Now using the above-mentioned canonical scalar product on $M_{2}(\mathbb{C})$, this gives the following formula for $P_{\alpha x \alpha}$, after cancelling the $(-1)^{2 N(\alpha)}=1$ term:

$$
\left\langle P_{\alpha x \alpha} \beta, \gamma\right\rangle=(-1)^{N(\alpha, \beta)+N(\alpha, \gamma)} x_{\beta} x_{\gamma} .
$$

Now summing over $\alpha$ gives the formula of the character $\chi(v)$ :

$$
\langle\chi(v) \beta, \gamma\rangle=\sum_{\alpha}(-1)^{N(\alpha, \beta)+N(\alpha, \gamma)} x_{\beta} x_{\gamma} .
$$

The coefficient of $x_{\beta} x_{\gamma}$ can be computed by using the multiplication table of quaternions,

$$
\sum_{\alpha}(-1)^{N(\alpha, \beta)+N(\alpha, \gamma)}=4 \delta_{\beta, \gamma}
$$

Thus $\chi(v)$ is a diagonal matrix, having the numbers $4 x_{\beta}^{2}$ on the diagonal:

$$
\chi(v)=\operatorname{diag}\left(4 x_{\beta}^{2}\right) .
$$

Consider the linear form $\varphi=\int \otimes t r$, where the integral is with respect to the Haar measure of $S U(2)$, and $t r$ is the normalised trace of $4 \times 4$ matrices, meaning $1 / 4$ times the usual trace. The moments of $\chi(v)$ with respect to $\varphi$ are computed as follows:

$$
\int \operatorname{tr}\left(\chi(v)^{k}\right) d x=4^{k-1} \sum_{\beta} \int x_{\beta}^{2 k} d x .
$$

By symmetry reasons the four integrals are all equal, say to the first one,

$$
\int \operatorname{tr}\left(\chi(v)^{k}\right) d x=4^{k} \int x_{1}^{2 k} d x .
$$

It follows that $\chi(v)$ has the same spectral measure as $4 x_{1}^{2}$,

$$
\mu_{\chi(v)}=\mu_{4 x_{1}^{2}} .
$$

But the variable $2 x_{1}$ is semicircular. This can be seen in many ways, for instance by direct computation, after identifying $S U(2)$ with the real sphere $S^{3}$, or by using the fact that $2 x_{1}=\operatorname{Tr}(x)$ is the character of the fundamental representation of $S U(2)$, whose moments are computed using Clebsch-Gordon rules. The result now follows by applying Theorem 2.1.

The construction of $\pi$ has the following generalisation. Consider the Clifford algebra $C l\left(\mathbb{R}^{s}\right)$. This is a finite-dimensional algebra, having a basis formed by products $e_{i_{1}} \ldots e_{i_{k}}$ with $1 \leq i_{1}<\ldots<i_{k} \leq s$, with multiplicative structure given by $e_{i}^{2}=-1$ and $e_{i} e_{j}=-e_{j} e_{i}$ for $i \neq j$.

It is convenient to use the notation $e_{I}=e_{i_{1}} \ldots e_{i_{k}}$ with $I=\left(i_{1}, \ldots, i_{k}\right)$.

As an example, the Clifford algebra $C l\left(\mathbb{R}^{2}\right)$ is spanned by the elements $e_{\emptyset}=1, e_{1}$, $e_{2}$ and $e_{12}=e_{1} e_{2}$. The generators $e_{1}, e_{2}$ are subject to the relations $e_{1}^{2}=e_{2}^{2}=-1$ and $e_{1} e_{2}=-e_{2} e_{1}$. Now these relations are satisfied by the Pauli matrices $i, j$, and the corresponding representation of $C l\left(\mathbb{R}^{2}\right)$ turns out to be faithful. That is, we have the following identifications:

$$
e_{\emptyset}=\left(\begin{array}{cc}
1 & 0 \\
0 & 1
\end{array}\right), \quad e_{1}=\left(\begin{array}{cc}
i & 0 \\
0 & -i
\end{array}\right), \quad e_{2}=\left(\begin{array}{cc}
0 & 1 \\
-1 & 0
\end{array}\right), \quad e_{12}=\left(\begin{array}{cc}
0 & i \\
i & 0
\end{array}\right) .
$$

We can label as well indices of $4 \times 4$ matrices by elements of the set $\{\emptyset, 1,2,12\}$. With these notations, the representation in Theorem 3.1 is given by $\pi\left(u_{I J}\right)=$ $P_{e_{I} x e_{J}}$. 
The same formula works for an arbitrary number $s$.

Theorem 3.2. There is a representation $\pi_{n}: A_{\text {aut }}\left(X_{n}\right) \rightarrow \mathbb{C}\left(G_{n}, M_{n}(\mathbb{C})\right)$ mapping the universal $n \times n$ magic biunitary matrix to the $n \times n$ matrix

$$
v=\left(P_{e_{I} x e_{J}}\right)_{I J}
$$

where $n=2^{s}$, the unitary group of the Clifford algebra $C l\left(\mathbb{R}^{s}\right)$ is denoted $G_{n}$, and the algebra of endomorphisms of $C l\left(\mathbb{R}^{s}\right)$ is identified with $M_{n}(\mathbb{C})$.

The first part of proof of Theorem 3.1 extends to this general situation. We get that $\chi(v)$ is diagonal, with eigenvalues $\left\{n x_{I}^{2}\right\}$. This does not seem to be related to semicircular elements when $s \geq 3$. The representation $\pi_{n}$ probably comes from an inner faithful representation of a quotient of $A_{a u t}\left(X_{n}\right)$, corresponding to a "subgroup" of the quantum permutation group.

\section{ACKNOWLEDGMENTS}

We are deeply grateful to Georges Skandalis for all the discussions and his essential contribution to the results of this paper.

\section{REFERENCES}

[1] E. Abe, Hopf algebras, Cambridge Univ. Press (1977). MR0594432 (83a:16010)

[2] T. Banica, Hopf algebras and subfactors associated to vertex models, J. Funct. Anal. 159 (1998), 243-266. MR.1654119 (2001a:46069)

[3] T. Banica, Symmetries of a generic coaction, Math. Ann. 314 (1999), 763-780. MR.1709109 (2001g:46146)

[4] T. Banica, Quantum automorphism groups of homogeneous graphs, J. Funct. Anal. 224 (2005), 243-280. MR2146039

[5] J. Bichon, Quantum automorphism groups of finite graphs, Proc. Amer. Math. Soc. 131 (2003), 665-673. MR 1937403 (2003j:16049)

[6] J. Kustermans and S. Vaes, Locally compact quantum groups in the von Neumann algebraic setting, Math. Scand. 92 (2003), 68-92. MR1951446 (2003k:46081)

[7] S. Vaes, Strictly outer actions of groups and quantum groups, J. Reine Angew. Math. $\mathbf{5 7 8}$ (2005), 147-184. MR2113893 (2005k:46167)

[8] D. Voiculescu, K. Dykema and A. Nica, Free random variables, CRM Monograph Series 1, AMS (1993). MR1217253(94c:46133)

[9] S. Wang, Quantum symmetry groups of finite spaces, Comm. Math. Phys. 195 (1998), 195211. MR:1637425 (99h:58014)

[10] S. Wang, Simple compact quantum groups I, preprint.

[11] S.L. Woronowicz, Compact matrix pseudogroups, Comm. Math. Phys. 111 (1987), 613-665. MR0901157 (88m:46079)

[12] S.L. Woronowicz, Tannaka-Krein duality for compact matrix pseudogroups. Twisted $S U(N)$ groups, Invent. Math. 93 (1988), 35-76. MR0943923 (90e:22033)

[13] S.L. Woronowicz, Compact quantum groups, in Symétries Quantiques - Les Houches 1995, North-Holland (1998), 845-884. MR1616348 (99m:46164)

Laboratoire Emile Picard, Université Paul Sabatier, 118 route de Narbonne, 31062 Toulouse, France

E-mail address: banica@picard.ups-tlse.fr

Institutul de Matematic ă al Academiei Române, P.O. Box 1-764, RO-014700 Bucureşti, ROMANIA

E-mail address: moroianu@alum.mit.edu 